\title{
IMPACTOS DA EXPLORAÇÃO FLORESTAL NA ESTRUTURA E NA COMPOSIÇÃO FLORÍSTICA DA VEGETAÇÃO REMANESCENTE EM ÁREA MANEJADA NA FLORESTA NACIONAL DO TAPAJÓS, BELTERRA, PARÁ
}

\author{
Maria Soraia Aguiar Castro; Ana Paula da Silva Viana²; Camila Carneiro Lobato3; Jackson Lyra Muniz4; \\ Max Weber dos Santos Lima ${ }^{5}$; Lia de Oliveira Melo.

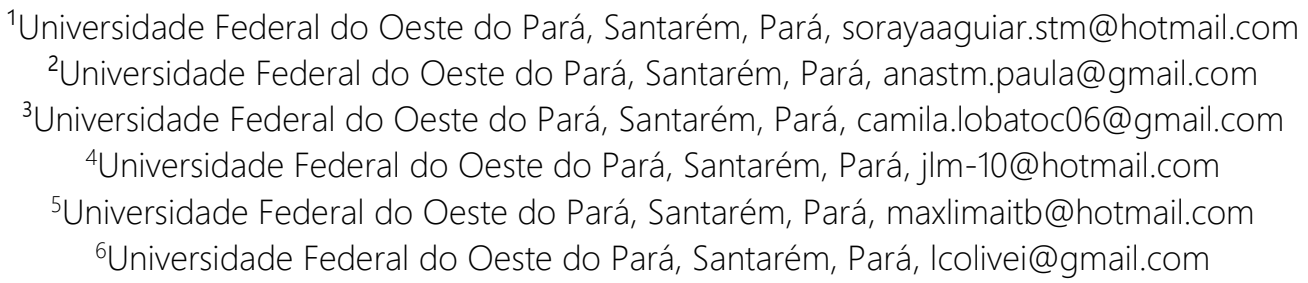

RESUMO: O presente trabalho foi desenvolvido em uma área de 1.600 ha situada na Floresta Nacional do Tapajós localizada em Belterra- Pará. Tem por objetivo, analisar os impactos, bem como, danos às árvores remanescentes, causados pela exploração madeireira proveniente do manejo comunitário na área. A amostra consistia em 8 parcelas permanentes (PP) de 0,25 hectares $(50 \mathrm{~m} \times 50 \mathrm{~m})$, divididas em 25 sub parcelas de $10 \mathrm{~m} \times 10 \mathrm{~m}$. Neste trabalho, foram realizadas mensurações das parcelas permanentes em dois momentos distintos, o primeiro antes de qualquer intervenção na floresta no ano de 2016 e, a segunda um ano após a intervenção para acompanhar o seu efeito, no ano de 2017. A diversidade florística manteve-se sem diferença significativa entre os anos avaliados variando de 153 para 150 espécies antes e após a exploração respectivamente. Destaca-se que os danos leves acometeram poucos indivíduos quando comparados com os que não sofreram dano algum e no que concerne à estrutura da floresta, esta foi pouco afetada conforme os índices de diversidade de Shannon-Wiener $\left(H^{\prime}\right)$ e o índice de equabilidade (E).

PALAVRAS CHAVE: Dinâmica de crescimento, Floresta Amazônica, Perturbação.

\section{IMPACTS OF FOREST EXPLOITATION ON THE STRUCTURE AND FLORISTIC COMPOSITION OF THE REMAINING VEGETATION IN AN AREA MANAGED IN THE FLORESTA NACIONAL DO TAPAJÓS, BELTERRA, PARÁ}

ABSTRACT: The present work was developed in an area of 1600 ha located in the Floresta Nacional do Tapajós, Belterra- Pará. Its objective is to analyze the impacts such as damage to remaining trees caused by logging from community management in the area caused by logging from community management in the area. The sample consisted of 8 permanent plots (PP) of 0.25 hectares $(50 \mathrm{~m} \times 50 \mathrm{~m})$, divided into 25 sub plots of $10 \mathrm{~m} \times 10 \mathrm{~m}$. In this work, measurements of the permanent plots were carried 
out at two different times, the first before any intervention in the forest in the year 2016 and the second one year after the intervention to follow its effect, in the year 2017. The floristic diversity maintained without significant difference between the evaluated years ranging from 153 to 150 species before and after the exploitation respectively. It should be noted that the mild damages affected few individuals when compared to those that did not suffer any damage and as regards the structure of the forest, this was little affected according to the diversity indices of Shannon- Wiener $\left(H^{\prime}\right)$ and the equability index (E).

KEYWORDS: Amazon rainforest, Disturbance, Growth dynamics.

\section{IMPACTOS DE LA EXPLOTACIÓN FORESTAL EN LA ESTRUCTURA Y COMPOSICIÓN FLORÍSTICA DE LA VEGETACIÓN REMANENTE EN ÁREA MANEJADA EN EL FLORESTA NACIONAL DO TAPAJÓS, BELTERRA, PARÁ}

RESUMEN: El presente trabajo fue desarrollado en un área de 1600 ha situada en el Floresta Nacional do Tapajós, Belterra- Pará. Tiene por objetivo, analizar los impactos causados por la explotación maderera como, daños a los árboles remanentes, causados por la explotación maderera proveniente del manejo comunitario en el área proveniente del manejo comunitario en el área. Entre las clases de tamaño, sólo se observó la clase de árbol. La muestra consistía en 8 parcelas permanentes (PP) de 0,25 hectáreas (50m x 50m), divididas en 25 subparcelas de $10 \mathrm{~m} \times 10 \mathrm{~m}$. En este trabajo se realizaron mediciones de las parcelas permanentes en dos momentos distintos, el primero antes de cualquier intervención en el bosque en el año 2016 y, la segunda un año después de la intervención para acompañar su efecto, en el año 2017. La diversidad florística mantuvo sin diferencia significativa entre los años evaluados que varían de 153 a 150 especies antes y después de la explotación respectivamente. Destaca-se que los daños leves y severos acometieron a pocos individuos cuando comparados con los que no sufrieron daño alguno y en lo que concierne a la estructura del bosque, ésta fue poco afectada conforme a las indigencias de diversidad de Shannon- Wiener ( $\left.\mathrm{H}^{\prime}\right)$ y el índice de equidad (E).

PALABRAS CLAVE: Dinámica de crecimiento, Perturbación, Selva amazónica.

\section{INTRODUÇÃO}

A grande maioria das florestas nativas da
Amazônia são comumente exploradas de forma não sustentável,

ignorando os critérios de sustentabilidade do manejo florestal, o 
que ocasiona uma drástica perda da cobertura vegetal, gerando uma diminuição de diversidade florística, sem que haja no entanto, o conhecimento da riqueza natural (SOUZA et al., 2006).

Desta forma, conhecer a estrutura da floresta se faz nos preceitos da colheita do plano de manejo florestal onde se julga o estágio de desenvolvimento da vegetação e se fomenta a aplicação de tratamentos silviculturais para as melhoras necessárias e produtividade da floresta (GAMA et al., 2007).

Diante disso, o estudo da dinâmica florestal leva em consideração o comportamento da floresta, onde a partir de mensurações, são calculadas as taxas de crescimento, dado pelo incremento em diâmetro; recrutamento ou ingresso, que é a entrada de novos indivíduos por meio da regeneração natural, e mortalidade, por causas naturais e sob manejo Azevedo et al., (2008). Essas informações podem ser obtidas através do procedimento conhecido como Inventário Florestal Contínuo (IFC), onde essas medições na floresta são repetidas em várias ocasiões (DE AZEVEDO et al., 2008).

Na tentativa de eliminar ou reduzir os danos causados pela colheita florestal, deve-se investigá-los cuidadosamente, revelando seus efeitos sobre a floresta remanescente com o objetivo de evitar os prejuízos nas produções futuras, apesar da regeneração natural após a colheita (MARTINS et al., 2003).

Para se conseguir uma boa colheita florestal, o Plano de Manejo Florestal (PMF) é essencial para que haja a produtividade associada a sustentabilidade. No entanto, mesmo com o planejamento adequado, a colheita provoca impactos inevitáveis a estrutura da floresta, e não garante 100\% a sustentabilidade da exploração Pinto, 2002). Destaca-se que, a estrutura e a composição florística são informações obtidas nos inventários florestais que se extraem ferramentas para a verificação da grande potencialidade da floresta e a estratégias para o manejo adequado (FRANCEZ et al., 2007).

Neste trabalho buscou-se avaliar o impacto da exploração florestal na 
composição florística, na estrutura de uma floresta nativa e na vegetação remanescente, em uma área submetida a exploração de impacto reduzido.

\section{MATERIAL E MÉTODOS}

A pesquisa foi realizada na Floresta Nacional do Tapajós, sendo esta uma unidade de conservação, localizada às margens do rio Tapajós e da BR-163, englobando áreas dos municípios de Belterra, Aveiro, Placas e Rurópolis, no estado do Pará, com aproximadamente 549.066,87 hectares. Tendo como coordenadas geográficas ou UTM: 2045 a $4^{\circ} 10^{\prime} \mathrm{S}$ e $54^{\circ} 45^{\prime}$, a $55^{\circ} 30^{\prime} \mathrm{W}$ e distante cerca $83 \mathrm{~km}$ do centro urbano do município de Santarém levando em consideração a base do IBAMA localizada no Km 83 da BR -163 (MMA, 2004).

O clima pode ser classificado como sendo tropical úmido com temperatura média anual de $25^{\circ} \mathrm{C}$, classificação é Ami, pelo sistema de Köppen com variação térmica anual inferior a $5^{\circ} \mathrm{C}$, apresentando um regime de chuvas bastante variado no decorrer do ano, quando comparado com de outras regiões do país. $\bigcirc$ solo da região pode ser classificado como sendo Latossolo Amarelo Distrófico em sua maioria (CORDEIRO, 2004).

Para Veloso et al. (1991) a vegetação predominante encontrada na região onde está situada a unidade de conservação pode ser classificada como Floresta Ombrófila Densa, tendo como uma de suas características a presença de árvores de grade porte.

O monitoramento de parcelas permanentes é feito através de parceria firmada entre a Universidade Federal do Oeste do Para (UFOPA) e a Cooperativa Mista da FLONA Tapajós (Coomflona), sendo este último, o responsável por manejar a áreas de produção de madeira. Os dados foram obtidos na Unidade de Produção Anual (UPA 11) de 1.600 hectares localizada no km 117 da BR-163, constituída por Unidades de Trabalhos (UT) de 100 hectares cada. A amostra consistiu em 8 parcelas permanentes (PP) de 0,25 hectares (50m x 50m), divididas em 25 sub parcelas de $10 \mathrm{~m} \times 10 \mathrm{~m}$. As parcelas permanentes 
foram instaladas com base no Manual de

Diretrizes de instalação e medição de parcelas permanentes proposto por (SILVA et al., 2005).

Foram mensuradas altura e DAP (Diâmetro à 1,30 m do solo). As variáveis observadas foram:

- $\quad$ Classe de identificação do fuste (CIF) que descreve os diversos estados em que podem ser encontradas as árvores em uma floresta;

- $\quad$ Situação silvicultural, identifica a situação em que se encontra cada árvore em relação às ações silviculturais aplicadas na floresta;

- $\quad$ Podridão, pode ser originada a partir de danos causados pela exploração, tratamentos silviculturais ou por agentes naturais;

- $\quad$ lluminação da copa, indica o nível de exposição da copa à luz, assim como o grau de competição existente com copas de árvores vizinhas;

- Forma de copa, é uma importante característica relacionada com o crescimento das árvores;

- $\quad$ Presença e efeito de cipós na árvore, é usada para estimar o grau de infestação dos cipós nas árvores monitoradas, e

- Danos, que avalia o estado de sanidade das árvores além de determinar a intensidade do impacto da exploração às árvores remanescentes e impacto de tratamentos silviculturais;

- Vento ou tempestade, devem ser observados tanto no fuste como na copa de árvores vivas (completas, quebradas ou caídas) com diâmetro $>10 \mathrm{~cm}$.

Essas observações foram anotadas em ficha de campo contendo outras informações como, número de árvore, código da espécie e nome comum.

As classes de tamanho, consideradas nas medições das parcelas permanentes foram: árvore (diâmetro > 10,0cm), arvoreta $(5,0 \mathrm{~cm}<$ diâmetro $<10,0 \mathrm{~cm})$, vara $(2,5 \mathrm{~cm}<$ diâmetro $<5,0 \mathrm{~cm})$ e muda (altura $>30 \mathrm{~cm}$ e diâmetro $<2,5 \mathrm{~cm}$ ). No entanto apenas a classe de árvore foi observada, haja vista que esta classe é mais comumente estudada.

Para este trabalho, as medições das parcelas permanentes ocorrem em dois momentos distintos. $\bigcirc$ primeiro antes de qualquer intervenção na floresta no ano 
de 2016 e, a segunda em 2017 após a intervenção, para melhor acompanhar o seu efeito. Destaca-se que as mensurações continuarão a ser realizadas ao longo do tempo.

Os dados coletados foram analisados e processados no programa Monitoramento de Florestas Tropicais (MFT) e Microsoft Excel versão 2013. Os parâmetros observados são referentes a danos, diversidade florística (Índice de Shannon) e riqueza florística, com o intuito de averiguar e analisar efeitos da exploração na unidade.

\section{RESULTADOS E DISCUSSÃO}

A riqueza florística foi analisada de acordo com a quantidade de famílias, gêneros e espécies contidos nas oito parcelas de estudo. Na Tabela 1 são demonstrados os dados obtidos nos dois anos de acompanhamento.

Para a mesma área no ano de 2017, após a colheita, nota-se que o número de famílias e gêneros permaneceu o mesmo variando apenas entre as parcelas, devido aos números de ingressos que mantiveram o equilíbrio na área. No entanto o número de espécies decaiu, ocorrendo a extinção de quatro espécies na área de estudo como Barbatimão (Stryphnodendron barbatiman Mart.), Caqui (Diospyros kaki), Escorrega-macaco (Vochysia haenkeana Mart.) e Guajará-bolacha (Syzygiopsis oppositifolia Ducke.) Ocorreu o surgimento de uma espécie nova a Murtinha (Blepharocalyx salicifolius Kunth $\bigcirc$. Berg).

O desaparecimento pode ter ocorrido em decorrência da existência de apenas um indivíduo representando cada uma das espécies citadas, além disso, elas se encontravam nas menores classes de tamanho que são as mais suscetíveis devido a sua fragilidade. Segundo Bulfe et. al (2009), em relação ao tamanho dos indivíduos danificados, há uma concentração nas classes diamétricas inferiores, o que afirma que eles estão distribuídos de acordo com a densidade relativa de cada uma das classes diamétricas. 
Tabela 1. Riqueza florística (genêro, família e espécie) em dois períodosna UPA tal na Floresta Nacional do Tapajós.

\begin{tabular}{ccccccccc}
\hline \multirow{2}{*}{ Parcela } & \multicolumn{3}{c}{2016 (antes da exploração) } & & \multicolumn{3}{c}{2017 (1 ano após exploração) } \\
\cline { 2 - 3 } & Família & Gênero & Espécie & & Família & Gênero & Espécie \\
\hline 1 & 22 & 35 & 44 & & 20 & 34 & 41 \\
2 & 21 & 39 & 54 & & 19 & 35 & 50 \\
3 & 20 & 24 & 37 & & 19 & 23 & 36 \\
4 & 28 & 45 & 59 & & 27 & 43 & 57 \\
5 & 28 & 41 & 57 & & 28 & 40 & 56 \\
6 & 24 & 33 & 45 & & 22 & 31 & 45 \\
7 & 25 & 34 & 43 & & 26 & 35 & 45 \\
8 & 25 & 37 & 51 & & 25 & 36 & 49 \\
\hline TOTAL & 43 & 94 & 153 & & 43 & 94 & 150 \\
\hline
\end{tabular}

Nisto, Leitão Filho (1987) ao analisar a riqueza florística de florestas subtropicais do Brasil, explanou que o número de famílias corrobora com o que se observa em áreas comumente amazônicas e consequentemente a quantidade de gêneros e espécies também. Isso mostra que a área de estudo pode representar bem outras áreas de floresta tropical.

Na Tabela 2 demonstra-se os danos leves causados por exploração (derruba e maquinário) que 28 árvores/ha sofreram, onde a classe de tamanho que foi mais afetada ficou entre 10 a 19,9 cm de DAP, com 7,5 indivíduos/ha que representam 53,5\% do total de árvores afetadas, visto que são as árvores menores e mais sensíveis do povoamento. Furtado (2009) afirmou que a extração da vegetação é relativamente baixa quando a mesma é planejada, o que não afeta de forma significativa a produção madeireira para uma futura colheita, visto que obedece a princípios do manejo florestal, de modo a garantir a conservação da floresta.

Silva et al. (2001) explanam em seu estudo no município de Mojú no Pará, onde $0,7 \mathrm{~m}^{3}$ por $\mathrm{m}^{3}$ foram extraídos, sendo que, para 1 árvore extraída, 19 sofreram danos. Já Gomes et al. (2004) encontraram na vegetação explorada, que $40,2 \%$ sofreram danos no tronco e na parte comerciável do indivíduo, o que pode vir a ser uma ameaça ao estoque da floresta remanescente. 
Tabela 2. Danos leves decorrentes da exploração, conforme densidade (N/ha), área basal (G) em $\mathrm{m}^{2} /$ ha e Volume $\left(\mathrm{m}^{3} / \mathrm{ha}\right)$ no ano de 2017.

\begin{tabular}{|c|c|c|c|c|c|c|}
\hline & \multirow{2}{*}{ Variável } & \multicolumn{5}{|c|}{ Classe de diâmetro (cm) } \\
\hline & & 10 a 19,9 & 20 a 29,9 & 30 a 39,9 & 70 a 79,9 & TOTAL \\
\hline \multirow{3}{*}{$\begin{array}{c}\text { Toda a } \\
\text { comunidade }\end{array}$} & N/há & 7,5 & 4,0 & 1,5 & 1,0 & 14,0 \\
\hline & $G\left(m^{2} / h a\right)$ & 0,1305 & 0,171 & 0,138 & 0,46 & 0,8995 \\
\hline & $V\left(m^{3} / h a\right)$ & 0 & 1,6 & 1,47 & 6,24 & 9,32 \\
\hline \multirow{3}{*}{ Total percentual } & N/há & 53,6 & 28,6 & 10,7 & 7,1 & 100 \\
\hline & $G\left(m^{2} / h a\right)$ & 14,5 & 19 & 15,3 & 51,1 & 100 \\
\hline & $V\left(m^{3} / h a\right)$ & & 17,2 & 15,8 & 67 & 100 \\
\hline $\begin{array}{c}\text { Total de } \\
\text { indivíduos }\end{array}$ & & 15 & 8 & 3 & 2 & 28 \\
\hline
\end{tabular}

No tangente a danos decorrentes da exploração da floresta, são vinculados ao planejamento da intensidade da atividade e de um extremo controle agregado à derruba, as operações que se entrelaçam como corte de cipó, mapeamento da área principalmente das árvores que foram extraídas, abertura de pátios e ramais de arraste, derruba, traçamento e derruba de fato foram planejadas metodicamente e o mais viável possível para reduzir os danos a vegetação remanescente (GOMES et al., 2006).

A partir do número de espécies é possível conhecer a diversidade da floresta, calculando índices como o de Shannon-Wiener $\left(H^{\prime}\right)$ e o índice de equabilidade (E), expressando a heterogeneidade florística da floresta (AMARAL et al., 2013).

Os valores de diversidade de espécies para toda a comunidade, assim como para cada parcela antes e após a exploração, calculada pelo índice de Shannon e índice de equabilidade estão apresentados na Tabela 3.

O índice de Shannon-Wiener para florestas tropicais varia de 3,83 a 5,85, valores considerados altos para qualquer tipo de vegetação (KNIGHT, 1975). Com isso, pode-se concluir que a área estudada apresenta grande diversidade florística, pois apresentou índice de 4,30 e 4,28 para toda a 
população na área antes e após exploração respectivamente. $\bigcirc$ grau estimado de equabilidade foi e 0,85 em toda comunidade para o ano de 2016 (antes da exploração) e 2017 (após a exploração), indicando alta uniformidade nas proporções do número de indivíduos/número de espécies existentes dentro da comunidade vegetal, resultados compatíveis foram obtidos por Gonçalves e Santos (2008) e Ximenes et al. (2011) em outros estudos conduzidos na Floresta Nacional do Tapajós. Em floresta de terra firme, valores aproximados foram encontrados por Francez et al. (2007) e da Silva Ribeiro et al. (2013), mostrando alta heterogeneidade das florestas localizadas na região amazônica.

Tabela 3. Parâmetros de diversidade, Shanon $(H)$, equabilidade $(E)$, para os anos de 2016 e 2017.

\begin{tabular}{cccc}
\hline & \multicolumn{3}{c}{2017} \\
\hline$H^{\prime}$ & $E$ & $H^{\prime}$ & \\
4,298288928 & 0,854456211 & 4,283404 & 0,854862 \\
\hline
\end{tabular}

*H'- Índice de diversidade de Shanon; E - Índice de equabilidade.

\section{CONCLUSÃO}

Os danos leves causados atingiram de forma mais acentuada os indivíduos que apresentam menor densidade, pois estes estão mais suscetíveis a danos, visto que são mais sensíveis a estes, equivalendo-se aos danos por causas naturais.

Desta forma, destaca-se que em sua maioria significativa, os indivíduos não sofreram danos, haja vista que o planejamento da exploração proporciona impactos reduzidos, bem como o constatado no presente estudo.

No que tange aos parâmetros de diversidade florística, observou-se que manteve-se a alta diversidade do local.

\section{REFERÊNCIAS}

AMARAL, L. D. P.; FERREIRA, R. A.; LISBOA, G. D. S.; Longhi, S. J.; 
WATZLAWICK, L. F. Variabilidade espacial do índice de diversidade de Shannon-Wiener em floresta ombrófila mista. Scientia Forestalis, Piracicaba, v. 42, n. 97, 2013.

AZEVEDO, C. P.; SANQUETTA, C. R.; SILVA, J. N. M.; MACHADO, S. A. Efeito de diferentes níveis de exploração e de tratamentos silviculturais sobre a dinâmica da floresta remanescente. Floresta, v. 38, n. 2, p. 277-293, 2008.

BULFE, N. M. L.; GALVÃO, F.; FIGUEIREDO FILHO, A.; MAC DONAGH, P. Efeitos da exploração convencional e de impacto reduzido em uma floresta estacional semidecidual na provincia de Misiones, nordeste da Argentina. Floresta, v. 39, n. 2, 2009.

CORDEIRO, A. Floresta Nacional do Tapajós: plano de manejo. Belterra: IBAMA, 2004.

SILVA RIBEIRO, R. B.; GAMA, J. R. V.; MARTINS, S. V.; MORAES, A.; DOS SANTOS, C. A. A.; CARVALHO, A. N. Estrutura florestal em projeto de assentamento, comunidade São Mateus, município de Placas, Pará, Brasil. Revista Ceres, Viçosa-MG, v. 60, n. 5, p. 610-620, 2013.

FRANCEZ, L. D. B.; CARVALHO, J. O. P.; JARDIM, F. D. S. Mudanças ocorridas na composição florística em decorrência da exploração florestal em uma área de floresta de Terra firme na região de Paragominas, PA. Acta Amazônica, VOL. 37(2), 219 - 228, 2007.
FURTADO, S. C. Dinâmica de uma floresta sob regime de manejo sustentável em escala empresarial na Amazônia Ocidental. Manaus: INPA/UFAM, 2009. 79 p.

GAMA, J. R. $\quad V_{\text {.; }}$ SOUZA, A. L.; CALEGÁRIO, N.; LANA, G. C. Fitossociologia de duas fitocenoses de floresta ombrófila aberta no município de Codó, estado do Maranhão. Revista Arvore, Viçosa-MG, v. 31, n. 3, p. 465477, 2007.

GOMES, A. P. C.; SOUZA, A. L. de; MEIRA NETO, J. A. A. Alteração estrutural de uma área florestal explorada convencionalmente na bacia do Paraíba do Sul, Minas Gerais, nos domínios de Floresta Atlântica. Revista Árvore, v. 28, n. 3, p. 407-417, 2004.

GONÇALVES, F. G; SANTOS, J. R. dos. Composição florística e estrutura de uma unidade de manejo florestal sustentável na Floresta Nacional do Tapajós, Pará. Acta Amazonica, v. 38, n. 2, p. 229-244, 2008.

KNIGHT, D. H. A Phytosociological analysis of species-rich tropical forest on barro colorado island, panama. Ecological monographs, $V$. 45, N. 3, P. 259-284, 1975.

LEITÃO FILHO, H. de F. Considerações sobre a florística de florestas tropicais e subtropicais do Brasil. IPEF, v. 35, p. 4146, 1987.

MARTINS, S. S.; COUTO, L.; MACHADO, C. C.; SOUZA, A. L. D. Efeito da exploração florestal seletiva 
em uma floresta estacional semidecidual. Revista Árvore, ViçosaMG, v. 27, n. 1, p. 65-70, 2003.

SILVA, S. M. A. da; SILVA, J. N. M.; BAIMA, A. M. V.; LOBATO, N. M.; THOMPSON, I. S.; COSTA FILHO, P. P. Impacto da exploração madeireira em floresta de terra firme no município de Moju, estado do Pará. In: SILVA, J. N. M.; CARVALHO, J. O. P. de; YARED, J. A. G. (Ed.) A silvicultura na Amazônia Oriental: contribuições do projeto Embrapa/DFID. Belém: Embrapa Amazônia Oriental: DFID, p. 309-323, 2001.

SILVA, J. N. M. Diretrizes para instalação e medição de parcelas permanentes em florestas naturais da Amazônia brasileira (No. 634.9209811 D598). Bélem, PA (Brasil): EMBRAPA Amazonia Oriental, Ministério da Agricultura, Pecuária e Abastecimento, Brasilia, DF, CIFOR, Belém Brasil, 2005.
SOUZA, R. S.; SOUZA, A. L.; LEITE, H. G.; YARED, J. A. G. Análise estrutural em Floresta Ombrófila Densa de terra firme não explorada, Amazônia Oriental. Revista Árvore. v. 30, p. 75-87, 2006.

VELOSO, H. P.; FILHO, A. L. R. R.; LIMA, J.C. A. Classificação da vegetação brasileira, adaptada a um sistema universal. Rio de Janeiro: Ministério da Economia, Fazenda e Planejamento, Fundação Instituto Brasileiro de Geografia e Estatística (IBGE), Diretoria de Geociências, Departamento de Recursos Naturais e Estudos Ambientais, 1991.

XIMENES, L.C.; VIEIRA, D.S.; GAMA, J.R.V.; SILVA-RIBEIRO, R.B.; CORREA, V.V.; ALVES, A.F. Estrutura de floresta manejada por comunitários na Flona Tapajós. SIMPÓSIO LATINOAMERICANO SOBRE MANEJO FLORESTAL, 5., Anais... Santa Maria: Universidade Federal de Santa Maria, 2011. p. 686-693. 